\title{
BMJ Open Engaging older people in an internet platform for cardiovascular risk self- management: a qualitative study among Dutch HATICE participants
}

\author{
Tessa van Middelaar, ${ }^{1,2}$ Cathrien R L Beishuizen, ${ }^{1}$ Juliette Guillemont, ${ }^{3}$ \\ Mariagnese Barbera, ${ }^{4}$ Edo Richard, ${ }^{1,2}$ Eric P Moll van Charante, ${ }^{5}$ on behalf of the \\ HATICE consortium
}

To cite: van Middelaar T, Beishuizen CRL, Guillemont J, et al. Engaging older people in an internet platform for cardiovascular risk selfmanagement: a qualitative study among Dutch HATICE participants. BMJ Open 2018;8:e019683. doi:10.1136/ bmjopen-2017-019683

- Prepublication history and additional material for this paper are available online. To view these files, please visit the journal online (http://dx.doi. org/10.1136/bmjopen-2017019683).

Received 19 September 2017 Revised 7 November 2017 Accepted 28 November 2017

Check for updates

${ }^{1}$ Department of Neurology, Academic Medical Center, Amsterdam, The Netherlands ${ }^{2}$ Department of Neurology, Donders Institute for Brain Cognition and Behaviour, Radboud University Medical Center, Nijmegen, The Netherlands

${ }^{3}$ Epidemiology and Public Health, University of Toulouse, Toulouse, France

${ }^{4}$ Institute of Clinical Medicine/ Neurology, University of Eastern Finland, Kuopio, Finland ${ }^{5}$ Department of General Practice, Amsterdam Public Health

Research Institute, Academic Medical Center, Amsterdam, The Netherlands

Correspondence to

Tessa van Middelaar; t.vanmiddelaar@amc.uva.nl

\section{ABSTRACT}

Objectives To study older peoples' experiences with an interactive internet platform for cardiovascular selfmanagement, to assess which factors influence initial and sustained engagement. To assess their views on future use within primary care.

Design Qualitative semistructured interview study, with thematic analysis.

Setting Primary care in the Netherlands.

Participants People $\geq 65$ years with an increased risk of cardiovascular disease who used the 'Healthy Ageing Through Internet Counselling in the Elderly' internet platform with remote support of a coach. Participants were selected using a purposive sampling method based on gender, age, level of education, cardiovascular history, diabetes, duration of participation and login frequency.

Results We performed 17 interviews with 20 participants, including three couples. In the initial phase, platform engagement was influenced by perceived computer literacy of the participants, user-friendliness, acceptability and appropriateness of the intervention and the initial interaction with the coach. Sustained platform use was mainly facilitated by a relationship of trust with the coach. Other facilitating factors were regular automatic and personal reminders, clear expectations of the platform, incorporation into daily routine, social support and a loyal and persistent attitude. Perceived lack of change in content of the platform could work both stimulating and discouraging. Participants supported the idea of embedding the platform into the primary care setting. Conclusions Human support is crucial to initial and sustained engagement of older people in using an interactive internet platform for cardiovascular selfmanagement. Regular reminders further facilitate sustained use, and increased tailoring to personal preference is recommended. Embedding the platform in primary healthcare may enhance future adoption.

Trial registration number ISRCTN48151589; Pre-results.

\section{INTRODUCTION}

In view of global ageing and the associated increasing burden of cardiovascular disease (CVD), prevention has become crucial. ${ }^{1}$ The
Strengths and limitations of this study

- We focused on the experiences of older people which is of increasing importance in view of global ageing.

- We iteratively adapted our interview guide to separately address influential factors on sustained engagement, as long-term adherence to lifestyle changes is a major challenge in cardiovascular prevention.

- Data collection with semistructured interviews and our purposive sampling method provided us with a broad view of people's experiences and provided insight into individual differences.

- We only interviewed Dutch people, potentially limiting the scope to the Dutch healthcare setting.

effectiveness of preventive interventions is indisputable, even in old age. ${ }^{2}{ }^{3}$ However, adherence to long-term lifestyle and medication regimens remains a daunting challenge. Average adherence rates for chronic illnesses are as low as 50\%. ${ }^{4}$ Currently, in several countries, cardiovascular risk management programmes are implemented into primary care and delivered by practice nurses. ${ }^{5}$ eHealth, that is, a method to deliver health services and information using the internet and related technologies, is a promising tool for delivery of prevention. ${ }^{6}$ It can enable self-management and improve the reach and sustainability of pre-existing preventive programmes. ${ }^{7}$ In particular, an eHealth platform combined with human support (ie, a blended approach) has shown beneficial effects on cardiovascular risk factors. ${ }^{8}$

Previous research on eHealth interventions identified several important influential factors of engagement; personal motivation, incorporation into personal life and quality of the eHealth intervention. ${ }^{9}$ However, it is unclear 
whether these are the same for initial and sustained engagement. For cardiovascular prevention, sustained engagement seems crucial, as the effectiveness of eHealth interventions on cardiovascular risk factors declines over time, especially after 1-year follow-up. ${ }^{8}$ Also, an eHealth intervention specifically targeted at older people should have a specific age-friendly design. ${ }^{10}$ It is important to assess the views of end users of an eHealth intervention to improve its chances of successful implementation. ${ }^{11} 12$

Our primary aim was to study older peoples' experiences with an interactive internet platform for cardiovascular self-management, to assess which factors influence initial and sustained engagement. Our secondary aim was to assess older people's views on implementation of such a platform in the primary care setting.

\section{METHODS}

\section{Setting and participants}

This qualitative study with semistructured interviews was performed among participants of the 'Healthy Ageing Through Internet Counselling in the Elderly' (HATICE, ISRCTN48151589) trial. ${ }^{13}$ HATICE is designed to investigate whether an internet platform for cardiovascular self-management can improve the cardiovascular risk profile. People $\geq 65$ years with an increased risk of CVD were recruited to participate in HATICE in the Netherlands, Finland and France. Computer illiteracy, defined as the inability to send an email, was an exclusion criteria for the trial. Through a thorough design and validation process, we developed the internet platform for cardiovascular self-management, adapted to meet the specific requirements of older people. ${ }^{10} 14$ The intervention is based on Bandura's social-cognitive theory for self-management and behaviour change and incorporated Michie's taxonomy for standardised definitions of behaviour change interventions. ${ }^{15} 16$ The platform offers blended care by remote support of a health-coach trained in motivational interviewing techniques and the transtheoretical (or stages of change) model. ${ }^{17} 18$ Participants can send messages and receive feedback from their coaches within the platform. Other functionalities of the platform include the ability to set lifestyle goals, record measurements (eg, blood pressure and weight), receive information on cardiovascular risk and healthy lifestyle and subscribe to lifestyle groups. The layout and navigation structure were kept simple to make the platform user-friendly for older people. The content was regularly updated with news items on relevant developments in cardiovascular prevention. The intervention was solely delivered via the platform, except for an initial inperson meeting with their coach at baseline, during which first lifestyle goals were set, and a phone call after 12 months follow-up.

This qualitative substudy was only performed among Dutch intervention participants. They were purposively sampled on gender, age, level of education, history of CVD, diabetes, duration of participation and login frequency.
Participants who prematurely ended their participation were also invited. Twenty out of 32 participants who were invited by telephone were willing to partake in the interview. Main reasons for people to decline participation were lack of time and too little overall use of the platform, even though we specifically aimed to also include these participants. All participants provided written informed consent.

\section{Data collection}

Between July 2016 and January 2017, three researchers (TvM, CRLB and Suzanne van Rhijn) held semistructured interviews following an interview guide (online supplementary appendix 1), focusing on participant experiences with the platform. We iteratively adapted the interview guide during the data collection period. For example, we decided to separately address initial and sustained use as distinct phases in the engagement and adoption of the intervention, as sustained engagement is especially challenging in lifestyle interventions. ${ }^{8}$ During the interviews, participants were asked to $\log$ onto the platform to stimulate the discussion. The final part of the interview guide focused on the interaction with regular care, during which participants were asked if they preferred the platform to be incorporated in primary healthcare. The interviewers all had experience with conducting qualitative interviews. Two of the interviewers (TvM and CRLB) were involved in the design and maintenance of the platform (the participants were not made aware of this) and one ( $\mathrm{SvR})$ in the logistical support of the trial. The interviewers and participants had no professional relationship prior to the interview. Participants were interviewed in private at their homes, and the interviews lasted approximately $50 \mathrm{~min}$. No repeat interviews were deemed necessary. Interviews were audiotaped and transcribed verbatim, and during the interviews, field notes were taken.

\section{Coding and analysis}

Two researchers (TvM and CRLB) thematically analysed the transcripts in an iterative process. ${ }^{19}$ First, each researcher independently coded transcripts following an inductive approach; next, the researchers discussed each other's codes to achieve interobserver agreement. Subsequently, the researchers together categorised the codes to generate a structure of main themes and subthemes. Themes were derived from the data and were not hypothesised prior to data collection. At several points during the analysis process, results were discussed with other team members to ensure independent interpretation. After the first seven interviews, the interview guide was adapted based on one of these discussions, leading to a better distinction between initial and sustained engagement with the platform. Questions about initial engagement were asked to all participants and about sustained engagement to participants who had been in the study for at least 6 months. After 17 interviews, data saturation was reached as no new (sub) themes or issues emerged. 
Table 1 Characteristics of the participants

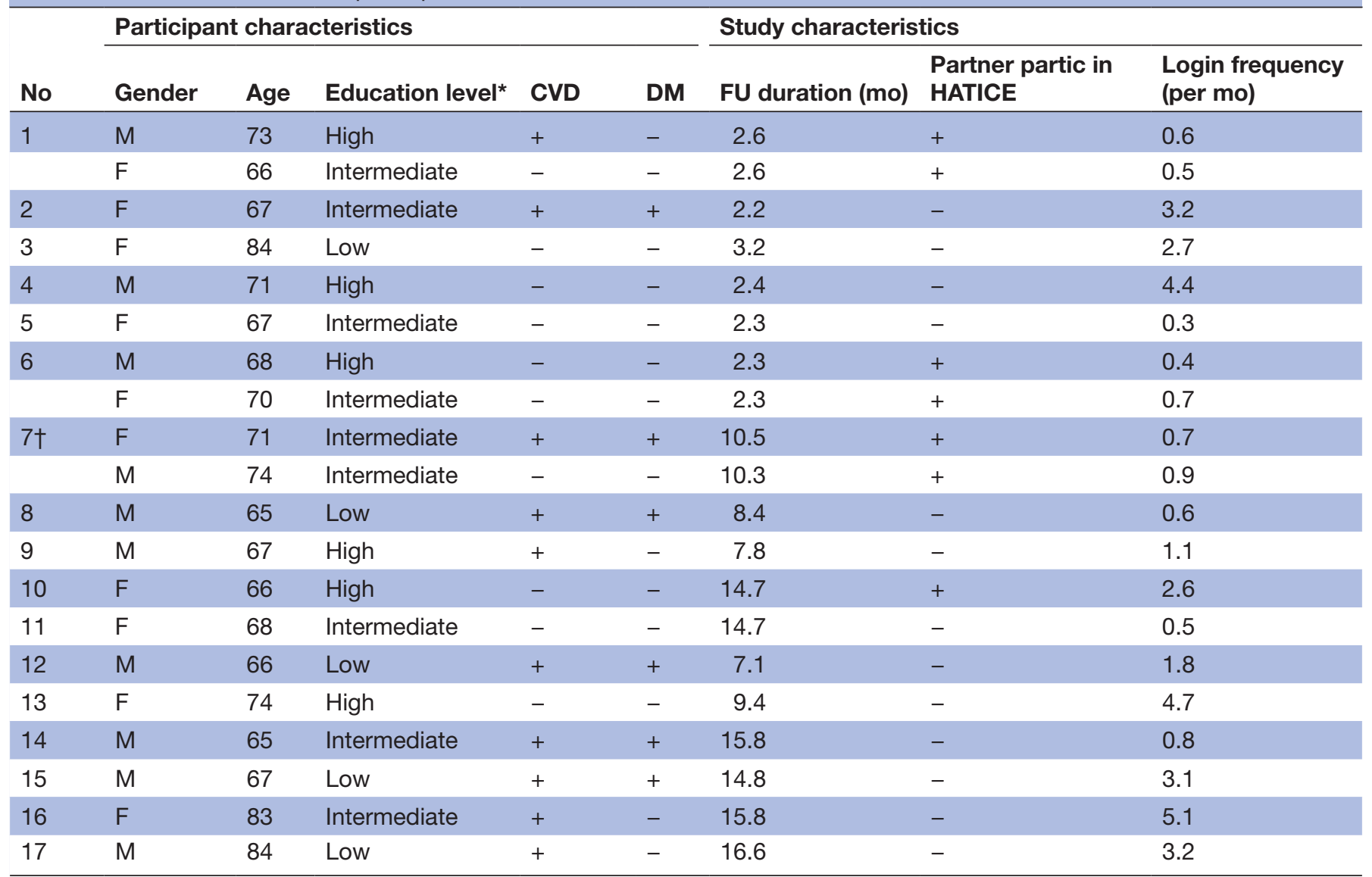

The characteristics are divided into participant characteristics and HATICE study characteristics.

*Low education level indicates primary education or lower secondary education; intermediate, upper secondary education and postsecondary non-tertiary education; high, short-cycle tertiary education.

†Interview seven was performed with participants who had recently (prematurely) ended their participation in HATICE.

+, yes; -, no; CVD, cardiovascular disease; DM, diabetes mellitus; F, female; FU, follow-up; HATICE, Healthy Ageing Through Internet

Counselling in the Elderly; M, male; mo, month; partic, participating.

\section{RESULTS}

We performed 17 interviews with 20 participants (table 1). Three interviews took place with couples participating in the HATICE trial together, one of which had prematurely dropped out from the trial. The age of the participants ranged from 65 to 84 years. Ten (50\%) participants had a history of CVD, and six (30\%) had diabetes. Length of participation in the trial ranged from short (2-3 months, $\mathrm{n}=8(40 \%))$, intermediate $(7-11$ months, $\mathrm{n}=6(30 \%))$ to long (14-17 months, $\mathrm{n}=6(30 \%))$. The main themes and subthemes of factors that influence initial and sustained platform engagement are presented in table 2 and further explained in the text below.

\section{Initial platform engagement}

User-friendliness for older people

Participants found the layout of the platform clear and simple which facilitated platform use. However, they stated that a more attractive platform could have encouraged them to $\log$ in more often:

You should have a website that makes you think, when you have some spare time at night or in the afternoon, why don't I just have a look at HATICE. [P8]

Technical difficulties in using the platform, for example, login difficulties, discouraged participants. Also, the notion of being inexperienced or incompetent with a computer or with the internet could hamper exploration of the platform and platform use. Sometimes, participants, together with their coach, found creative ways to use the platform when this was considered difficult:

I'm not a computer freak. [...] Once I receive a message then I answer it. And then she [coach] says, you should also complete it in the category that it belongs to [measurement]. To me it is not easy to find that [...] But then later I notice that she has neatly entered it [in the measurement functionality]. I think that's fine. [P12]

People who regarded themselves as inquisitive or eager to learn said this stimulated them in exploring the different functionalities of the platform. 
Table 2 Themes and subthemes identified in the interviews of the facilitators $(+)$ and barriers $(-)$ in initial and sustained platform use

\begin{tabular}{ll}
\hline Initial platform use & Sustained platform use \\
\hline User-friendliness for older people & Coach: long-term relationship of trust \\
- Layout: simplicity, attractiveness $(+/-)$ & Personal connection $(+)$ \\
- Technical difficulties $(-)$ & Content of messages $(+/-)$ \\
Perceived computer literacy $(+/-)$ & Continuity of person $(-)$ \\
Coach: the basis for a relationship of trust & Reactive use of the platform* $(+)$ \\
- Inperson baseline consultation $(+)$ & Lifestyle change: expectations and experiences \\
- Timing and content of messages $(+)$ & - Expectations of platform $(+/-)$ \\
Usefulness and perceived benefit of the intervention & Benefits of lifestyle changes $(+)$ \\
- Affinity with platform functionalities (including self- & Setting a goal is burdensome $(-)$ \\
management) $(+/-)$ & Monitoring health $(+)$ \\
Awareness of cardiovascular risk $(+/-)$ & Incorporation into daily routine \\
- Motivation for lifestyle change with increasing age $(-)$ & Incorporation into daily routine $(+/-)$ \\
& Social (partner) support $(+)$ \\
& - Continuity of care $(+/-)$ \\
& Pime investment $(+/-)$ \\
& Perceived lack of change in the platform $(+/-)$ \\
\hline
\end{tabular}

${ }^{*}$ Reactive use indicates the preference of participants to use the platform in response to automatic or personal reminders.

Coach: the basis for a relationship of trust

For participants, trusting the coach was a prerequisite to talk about their health behaviours and potential lifestyle goals. The inperson baseline consultation with their coach was much appreciated and formed a basis to build a relationship of trust. If the coach responded quickly and adequately to messages sent after the baseline visit, this stimulated platform engagement:

At first I wanted... I really had no... I mean, I was actually curious. I did not think well this will... for me... At a certain moment, also because of her [coach], I immediately received a message back and she stimulated me, she said 'oh well done' and I don't know what more. That made me say, OK I will continue with this. [P12]

Instead, if messages were not answered timely, participants became discouraged to continue using the platform. Some participants found personal contact through the messaging system insufficient to build a relationship and missed face-to-face or telephone contact.

\section{Usefulness and perceived benefit of the intervention}

During their first encounter with the platform, participants tended to focus on a small number of functionalities that appeared useful and relevant and continued with these over time. This mostly concerned the messaging and measurement functionalities:

When I receive an email I will go to the website and $\log$ in. And then I see what happened [message] and have a look. And sometimes I'm asked to complete a questionnaire and I do that. And other times, as is the case now, I'll go to the practice nurse; well then I have my blood and urine tested, and I send those along [send results to the coach]. [P16]
Some participants reported affinity with self-management and self-measuring of cardiovascular risk factors. They perceived the measurement functionality as useful and appropriate, facilitating platform use. Conversely, limited affinity with self-management could form a barrier to use this functionality:

And I absolutely do not want my own blood pressure monitor. I did not want that when it [blood pressure] was too high and I certainly do not want it now that it is too low. Because I get very uh... It will influence me and I don't want that. I will not make myself crazy. [P3]

Participants who were aware of their cardiovascular risk status, in some cases because of a previous CVD, deemed the content of the platform relevant. Participants with limited perceived need to improve their lifestyle did not see how the platform could help them and tended to make limited use of it:

I notice that it's about CVD. That is all fine, but I don't have that [history of CVD], so I will not engage any further with it [the platform]. [...] Indeed, if I do encounter it [CVD], than I would do it, but at this moment... [P5]

Participants who already frequently visited their healthcare professional(s) stated they did not expect important additional benefit. Age also played a role as one of the oldest participants no longer prioritised adapting a healthier lifestyle because of his old age. Participants rarely adjusted or replaced the goals that were set at baseline. Limited use was made of the suggestions for lifestyle groups; participants expressed several reservations related to this functionality, such as that they thought that signing up created an obligation to participate and that 
groups would be dominated by older people with very limited functionalities.

\section{Sustained platform engagement Coach: long-term relationship of trust}

As mentioned above, the coach was important to stimulate initial use of the platform. The coach also appeared pivotal in sustained platform use. If participants felt connected to the coach, participants felt inclined to keep using the platform and adhere to goals for lifestyle changes:

Yes, because the coach makes you try to accomplish certain things. [...] That would be more difficult without the coach. I don't know if... every time with the website... no, I don't think that that would work on its own [platform without coach]. [P9]

The message content was also important; a positive and personal tone could boost someone's motivation. One interviewee had experienced a change in coach during the trial. He stated this did not clearly change his platform use, although it did negatively impact his connection with the coach.

\section{Reactive use of the platform}

In many interviews, participants expressed difficulty to take initiative in using the platform and found it easier to use the platform in a reactive way, for example, responding to automatic or personal reminders:

Look, I like to participate in such a study, but... Perhaps I'm a bit more passive, that I think even if I have to have ten visits a year, that is fine. We will have a conversation; I will complete lists; that is all fine. But a website is... to figure things out, and to write things down, that is something... [Interviewer: Maybe you can call that initiative?] Yes I suppose that could be it. [P14]

Participants who considered themselves as being loyal or persistent noted this stimulated sustained platform use:

I was told to make contact once a month. And so I... It's stated here in my iPad: remember HATICE, report! And so we plan to do that. [P11]

\section{Lifestyle change: expectations and experiences}

Being motivated for lifestyle change was a reason to continue using the platform and vice versa. This could be related to the reason to participate in the HATICE trial. Some participants were aware that the trial entailed active participation and hoped that they might benefit from it. Others, who participated to contribute to scientific progress, seemed to expect a more passive participation; that is, questionnaires or tests for which no self-initiative was required, and were not inclined to use the platform for self-management. Second, if people managed to reach their lifestyle goals and experienced its positive effects on their health, this stimulated sustained participation:
Five kilometre laps. Yes, that is the minimum distance that I would like to walk each time. And I can achieve that quite nicely. And in that, I noticed that I started to feel fitter. That was really surprising. I always thought that I would stumble along through the rest of my life. And now I can... you get more fit. You have more enthusiasm to tackle things. [P14]

In contrast, some participants felt setting a goal was an unpleasant burden. If they did not manage to reach their goal, they refrained from registering this on the platform or informing their coach, also, because they felt embarrassed or demotivated:

You got sort of forced to... Because you had to make certain promises, like 'I will make sure to exercise so many times a day' and 'I will make sure I will lose weight'. Those kinds of things. Yes, that went against my gut feeling. [...] You were sort of embarrassed if you said, well I actually did not do anything. [P7]

Participants appreciated the automatic feedback on entered measurements as it gave a reassuring feeling of having their health monitored. This facilitated regular logging in.

\section{Incorporation into daily routines}

Participants said that it was easier for them to keep using the platform if they had incorporated their platform use into their daily or weekly routine:

Yes I like it. It works as a sort of support. In life you have all kinds of support systems, with your habits and your things, and this is one of them. It has become a part of... Yes well sometimes I can use it and sometimes I can't. But it has become a part of everything. [P2]

Disruption of daily routines, such as illnesses, negatively affected platform use. Social support, on the other hand, was an incentive for sustained use. This was especially true for couples participating in the HATICE trial together:

I said, 'We should do something.' Then I started to fill those [questionnaires] in. And I said, 'Are you going to do that?' [Response partner:] 'Yes I will do that, but I am very busy.' I said, 'It will only take a minute.' [P10]

Another important factor that facilitated platform-adherence was that the platform could improve the perceived continuity of support in self-management. In contrast to nurse-led periodic consultations, which are typical of secondary cardiovascular prevention programmes, the platform felt like a source of continuous support that they could direct to any time:

I already visit the practice nurse, but there is a lot of time in between [visits] and then yes... Of course together we assess the results, look at it and discuss it. But when I'm gone, it [the support] is also gone. 
Unless, of course, it turns out that I have to... that it's not quite OK. But then it's gone again. And this is, the continuity that you're always working on it, that is good. [P2]

Some participants found using the platform was time-consuming, which worked as a barrier. This could occur because of the misconception that they were obliged to regularly add measurements. In contrast, if participants felt the platform did not take too much of their time, they were inclined to keep using it.

\section{Perceived lack of change in the platform}

Most participants were not aware of any changes made to the platform content, although others noted that news items were regularly updated. While several participants appreciated the stable content, others would have liked to see more changes over time, to stimulate their sustained engagement:

Well I read that [information on cardiovascular risk] a little in the beginning and then that is that. Well now... And that does not change. I'm almost certain that this is the same as it was 1,5 year ago. [...] So that is not inviting; to keep looking if there is something new. $[\mathrm{P} 15]$

The coach could influence this by varying the themes of conversation.

\section{Future implementation}

Participants indicated that the level of incorporation into the regular healthcare system was limited, and therefore, some of them felt the platform had no clear added value on top of the nurse-led cardiovascular risk management they already received within the primary healthcare. Regarding future implementation, participants felt positive towards incorporation of the platform into the existing primary care structure. Especially if the practice nurse were to become their coach, thus contributing to continuity of support, and if all measurements performed at home, and within primary and secondary care were integrated into the platform:

The visit to the practice nurse is of course the real measurement. So I feel it's important to keep that, because it monitors your health, or at least a part of your health. That is important. But if all those measurements could be incorporated into this study, that would of course be very positive, because than you can compare it over several years or you can use it to look things up. [P9]

A concern of some of the participants was that this incorporation would lead to substitution of valued, inperson contacts with healthcare professionals by more anonymous exchange of messages via the platform. A participant suggested to add regular inperson visits with measurements to increase motivation, as a solution.

\section{DISCUSSION}

\section{Summary}

We have found that the support of a coach is crucial to initiate and sustain engagement of older people with an interactive internet platform for cardiovascular self-management. Factors associated with initial platform engagement are perceived computer literacy, usability and anticipated benefits of the platform, with special attention to the computer skills and preferences of older people. Factors associated with sustained platform engagement are regular automatic and personal reminders, clear expectations, incorporation into daily routine and social support. Incorporation into primary healthcare could facilitate implementation of the platform and could improve the perceived continuity of support in self-management.

\section{Strengths and limitations}

The main strength of our study is that through our purposive sampling method, we included participants with short, intermediate and long follow-up durations. This contributed to a clear distinction in motives for initial and sustained engagement. We used an iterative analysis method with multiple analysis rounds and adaptation of the interview guide throughout the process. Also, we followed the consolidated criteria for reporting qualitative research guidelines to facilitate reproducibility of study results. ${ }^{20}$ A limitation of our study is that we only interviewed Dutch participants, potentially limiting the scope to the Dutch healthcare setting. Furthermore, the sample is prone to bias as our participants were willing to partake in both the HATICE trial and our qualitative substudy. This could have led to selection of people with a relative positive view on the intervention and with a high education level. ${ }^{21}$ We minimised this potential bias by purposively sampling participants on education level and login frequency. Another possible source of bias is the fact that two of the interviewers and researchers analysing the data were involved in the development and maintenance of the platform. This could have influenced the intonation of questioning and interpretation of the data; however, their knowledge of the platform could also have stimulated the discussion. Independent analysis was ensured by incorporation of several analysis rounds with other team members.

\section{Comparison with existing literature}

Part of our results are in line with previous studies on engagement with eHealth interventions, such as on the influence of usability, perceived benefit and expectations of the intervention and the incorporation into personal life. ${ }^{9}$ A new finding that is especially relevant for eHealth interventions on cardiovascular prevention is the crucial role of continuous support by a coach for sustained engagement. This has previously been described in a non-digital multidomain preventive intervention. ${ }^{22}$ In our study, the initial inperson contact was important to establish a relationship of trust between the participant and coach. For most 
people, maintenance of this relationship via a messaging system appeared to work well for a long-standing personal connection. The importance of this kind of blended care is emphasised by a meta-analysis showing a more pronounced effect on cardiovascular risk reduction. ${ }^{8}$ Despite the use of motivational interviewing techniques and coaches following the transtheoretical model, ${ }^{17}{ }^{18}$ it was difficult to engage people with a low perceived benefit of the intervention. In general, motivational interviewing techniques delivered through eHealth have proven effective in inducing behavioural changes. ${ }^{23}$ Nevertheless, a complete inperson approach might be preferable for participants in the precontemplation phase, when there is no intention to change behaviour, as even reading information about cardiovascular risk on the platform requires some level of initiative. ${ }^{15}$ A reactive approach, that is, responding to automatic and personal reminders, rather than a proactive approach seemed to suit most participants best. Previous studies have shown that electronic reminders are a useful tool to increase medication adherence. ${ }^{24}$ However, it is uncertain whether this reactive approach sufficiently supports self-efficacy. ${ }^{15}$ In line with the degrees of self-management proposed by Schermer, this might be seen as compliant self-management. ${ }^{25}$ Even though the interactive and flexible quality of the HATICE platform facilitates adoption of concordant self-management, that is, incorporation of the lifestyle advice into their personal life, this is not employed by everyone. Limited computer experience is an important barrier to platform use which may prohibit large-scale implementation. Increasing use of internet by older people is likely to overcome this limitation in the near future. ${ }^{26}$

\section{A tailored platform}

Our study shows that many aspects of multidomain eHealth interventions rely heavily on personal preferences. The HATICE platform has been adjusted to the need for a personalised platform, by not imposing any obligations on which functionalities to use and giving participants the opportunity to tailor the frequency of automatic reminders to personal preferences. However, during the interviews, it appeared that people prefer an even more personalised platform. For instance, engagement was dependent on personal preference with regard to how much the content of the platform changes over time and the complexity of the platform changes, affinity with self-measurement, whether or not confrontation with lifestyle goals was appreciated, the ideal amount of time invested and the optimal frequency of reminders. As suggested by Bandura, it might be useful to tailor the platform content and the way it is provided based on a participant's readiness to change. ${ }^{15}$ This could, for example, be incorporated in a self-learning system that automatically tailors to personal characteristics, stages of change, needs and wishes. ${ }^{27}$

\section{Implications for practice}

During the HATICE trial, the platform was offered independently from regular care. Participants mentioned this separation as a barrier to platform use and agreed with the suggestion to incorporate it into the current primary care structure. Preventive eHealth interventions provide the opportunity to optimise continuity in support of self-management and reach individual targets with limited resources. In addition, implementation may improve sustained engagement with such an intervention. ${ }^{28}$ Suggestions for this incorporation are to have the practice nurse work as coach, link measurements from electronic health records directly to the platform and align this with additional inperson visits for nurse-led cardiovascular risk management. Nevertheless, opportunities to implement the platform probably differ based on the healthcare system. It is therefore crucial to properly evaluate the healthcare context and views of end users and healthcare professionals to support successful implementation. ${ }^{12}$ Especially in healthcare systems with long distances or low resources, a preventive eHealth intervention may provide opportunities to improve existing preventive care. ${ }^{29}$

Acknowledgements We would like to thank all HATICE participants who agreed to participate in this qualitative substudy. Our gratitude also goes out to Suzanne van Rhijn for her help in interviewing participants and Sinem Kurt-Bayrakci for transcribing part of the interviews.

Collaborators Academic Medical Center (AMC), Amsterdam, The Netherlands: ER, Willem A. van Gool, EPMvC, CRLB, Susan Jongstra, TvM, Lennard van Wanrooij and Marieke Hoevenaar-Blom; University of Eastern Finland (UEF), Kuopio, Finland: Hilkka Soininen, Tiia Ngandu and MB; Karolinska Institutet/Stockholm University, Stockholm, Sweden: Miia Kivipelto and Francesca Mangialasche; INSERM, University of Toulouse, Toulouse, France: Sandrine Andrieu, Nicola Coley and JG; NOVAPTEN, Paris, France: Yannick Meiller; VitalHealth Software, Ede, The Netherlands: Bram van de Groep; Cambridge Institute of Public Health, University of Cambridge, Cambridge, UK: Carol Brayne.

Contributors TVM and CRLB: study design, data acquisition, data analysis, data interpretation and drafting of the manuscript. JG and MB: data interpretation and critical revision of the manuscript. ER and EPMvC: study design, data interpretation and critical revision of the manuscript.

Funding The HATICE trial is funded by the European Union Seventh Framework Programme (FP7/2007-2013) under grant agreement no 305374. TvM was supported by an EU Joint Programme-Neurodegenerative Disease Research (JPND) project. The project is supported through the following funding organisations under the aegis of JPND-www.jpnd.eu: Finland, Suomen Akatemia (Academy of Finland, 291803); France, L'Agence Nationale de la Recherche (The French National Research Agency, ANR-14-JPPS-0001-02); Germany, Bundesministerium für Bildung und Forschung (BMBF) (The Federal Ministry of Education and Research, FKZ01ED1509); Sweden, Vetenskapsrådet (VR) (Swedish Research Council, 529-2014-7503); The Netherlands, ZonMw (The Netherlands Organisation for Health Research and Development, 733051041).

Competing interests None declared.

Patient consent Obtained.

Ethics approval Medical Ethics Committee of the Academic Medical Center.

Provenance and peer review Not commissioned; externally peer reviewed.

Data sharing statement Data will not be made publicly available. For more information, please contact the corresponding author.

Open Access This is an Open Access article distributed in accordance with the Creative Commons Attribution Non Commercial (CC BY-NC 4.0) license, which permits others to distribute, remix, adapt, build upon this work non-commercially, and license their derivative works on different terms, provided the original work is properly cited and the use is non-commercial. See: http://creativecommons.org/ licenses/by-nc/4.0/

(c) Article author(s) (or their employer(s) unless otherwise stated in the text of the article) 2018. All rights reserved. No commercial use is permitted unless otherwise expressly granted. 


\section{REFERENCES}

1. Abegunde DO, Mathers CD, Adam T, et al. The burden and costs of chronic diseases in low-income and middle-income countries. Lancet 2007;370:1929-38.

2. Mons U, Müezzinler A, Gellert C, et al. Impact of smoking and smoking cessation on cardiovascular events and mortality among older adults: meta-analysis of individual participant data from prospective cohort studies of the CHANCES consortium. BMJ 2015;350:h1551.

3. Chow CK, Jolly S, Rao-Melacini P, et al. Association of diet, exercise, and smoking modification with risk of early cardiovascular events after acute coronary syndromes. Circulation 2010;121:750-8.

4. World Health Organization. Adherence to long-term therapies: evidence for action, 2003. http://www.who.int/chp/knowledge/ publications/adherence report/en/ (accessed 17 Dec 2017).

5. Nouwens E, van Lieshout J, van den Hombergh P, et al. Shifting cardiovascular care to nurses results in structured chronic care. Am J Manag Care 2014;20:e278-84.

6. Pagliari C, Sloan D, Gregor P, et al. What is eHealth (4): a scoping exercise to map the field. J Med Internet Res 2005;7:e9.

7. Kohl LF, Crutzen R, de Vries NK. Online prevention aimed at lifestyle behaviors: a systematic review of reviews. J Med Internet Res 2013;15:e146.

8. Beishuizen CR, Stephan BC, van Gool WA, et al. Web-based interventions targeting cardiovascular risk factors in middle-aged and older people: a systematic review and meta-analysis. J Med Internet Res 2016;18:e55.

9. O'Connor S, Hanlon P, O'Donnell CA, et al. Understanding factors affecting patient and public engagement and recruitment to digital health interventions: a systematic review of qualitative studies. BMC Med Inform Decis Mak 2016;16:120.

10. Becker SA. A study of web usability for older adults seeking online health resources. ACM Trans Comput Hum 2004;11:387-406.

11. Peters $\mathrm{DH}$, Adam $\mathrm{T}$, Alonge $\mathrm{O}$, et al. Implementation research: what it is and how to do it. BMJ 2013;347:f6753.

12. Glasgow RE, Phillips SM, Sanchez MA. Implementation science approaches for integrating eHealth research into practice and policy. Int J Med Inform 2014;83:e1-11.

13. Richard E, Jongstra S, Soininen $\mathrm{H}$, et al. Healthy Ageing Through Internet Counselling in the Elderly: the HATICE randomised controlled trial for the prevention of cardiovascular disease and cognitive impairment. BMJ Open 2016;6:e010806.

14. Jongstra S, Beishuizen C, Andrieu S, et al. Development and validation of an interactive internet platform for older people: The
Healthy Ageing Through Internet Counselling in the Elderly Study. Telemed J E Health 2017;23:96-104.

15. Bandura A. Health promotion by social cognitive means. Health Educ Behav 2004;31:143-64.

16. Michie S, Richardson M, Johnston M, et al. The behavior change technique taxonomy ( $v 1$ ) of 93 hierarchically clustered techniques: building an international consensus for the reporting of behavior change interventions. Ann Behav Med 2013;46:81-95.

17. Rollnick S, Miller WR, Butler CC. Motivational interviewing in health care: helping patients change behavior. New York: The Guilford Press, 2008.

18. Prochaska JO, DiClemente CC, Norcross JC. In search of how people change. Applications to addictive behaviors. Am Psychol 1992;47:1102-14.

19. Braun V, Clarke V. Using thematic analysis in psychology. Qual Res Psychol 2006;3:77-101.

20. Tong A, Sainsbury P, Craig J. Consolidated criteria for reporting qualitative research (COREQ): a 32-item checklist for interviews and focus groups. Int J Qual Health Care 2007;19:349-57.

21. Rothwell PM. External validity of randomised controlled trials: "to whom do the results of this trial apply?". Lancet 2005;365:82-93.

22. Ligthart SA, van den Eerenbeemt KD, Pols J, et al. Perspectives of older people engaging in nurse-led cardiovascular prevention programmes: a qualitative study in primary care in The Netherlands. Br J Gen Pract 2015;65:e41-8.

23. Shingleton RM, Palfai TP. Technology-delivered adaptations of motivational interviewing for health-related behaviors: A systematic review of the current research. Patient Educ Couns 2016;99:17-35.

24. Vervloet M, Linn AJ, van Weert JC, et al. The effectiveness of interventions using electronic reminders to improve adherence to chronic medication: a systematic review of the literature. J Am Med Inform Assoc 2012;19:696-704.

25. Schermer M. Telecare and self-management: opportunity to change the paradigm? J Med Ethics 2009;35:688-91.

26. Eurostat. Internet access and use statistics - households and individuals. http://ec.europa.eu/eurostat/statistics-explained/ index.php/Internet_access_and_use_statistics_-_households_and_ individuals (accessed 24 Apr 2017).

27. Conway N, Webster C, Smith B, et al. eHealth and the use of individually tailored information: a systematic review. Health Informatics J 2017;23:218-33.

28. Brown LL, Lustria ML, Rankins J. A review of web-assisted interventions for diabetes management: maximizing the potential for improving health outcomes. J Diabetes Sci Technol 2007;1:892-902.

29. Saner $\mathrm{H}$, van der Velde $\mathrm{E}$. eHealth in cardiovascular medicine: clinical update. Eur J Prev Cardiol 2016;23:5-12. 